\title{
Generic bifurcation of Hamiltonian vector fields with symmetry
}

\author{
Michael Dellnitz $\nmid \S$, Ian Melbourne $\uparrow \|$ and Jerrold E Marsden $\uparrow q$ \\ + Department of Mathematics, University of Houston, Houston, TX 77204-3476, USA \\ $\ddagger$ Institut für Angewandte Mathematik, Universität Hamburg, W-2000 Hamburg 13, Fed- \\ eral Republic of Germany
}

Received 19 March 1992

Accepted by D A Rand

\begin{abstract}
One of the goals of this paper is to describe explicitly the generic movement of eigenvalues through a one-to-one resonance in a linear Hamiltonian system which is equivariant with respect to a symplectic representation of a compact Lie group. We classify this movement, and hence answer the question of when the collisions are 'dangerous' in the sense of Krein by using a combination of group theory and definiteness properties of the associated quadratic Hamiltonian. For example, for systems with no symmetry or $O$ (2) symmetry generically the eigenvalues split, whereas for systems with $S^{1}$ symmetry, generically the eigenvalues may split or pass. It is in this last case that one has to use both group theory and energetics to determine the generic eigenvalue movement. The way energetics and group theory are combined is summarized in table 1 . The result is to be contrasted with the bifurcation of steady states (zero eigenvalue) where one can use either group theory alone (Golubitsky and Stewart) or definiteness properties of the Hamiltonian (Cartan-Oh) to determine whether the eigenvalues split or pass on the imaginary axis.
\end{abstract}

AMS classification scheme numbers: $58 \mathrm{~F} 05,58 \mathrm{~F} 14$

\section{Introduction}

Hamiltonian vector fields can undergo a variety of bifurcations as a single bifurcation parameter is varied (see Abraham and Marsden [1]). Consider the following two types of local bifurcation from an equilibrium:

(i) steady-state bifurcation when the linearized vector field at the equilibrium has a zero eigenvalue of multiplicity two; and

(ii) 1-1 resonance when the linearization has a pair of purely imaginary eigenvalues of multiplicity two;

Without loss of generality, we may assume in case (ii) that these eigenvalues are $\pm \mathrm{i}$.

Let $\omega$ denote the symplectic form. In case (i), the kernel of the linearization is a two-dimensional symplectic subspace. As the bifurcation parameter is varied,

$\S$ Research supported by the Deutsche Forschungsgemeinschaft and by NSF/DARPA DMS-8700897. Permanent address: Institut für Angewandte Mathematik, Universität Hamburg, D-2000 Hamburg 13, Federal Republic of Germany.

|| Supported in part by NSF/DARPA DMS-8700897.

$\uparrow$ Research partially supported by a Humboldt award. Permanent address: Department of Mathematics, University of California at Berkeley, Berkeley, CA 94720, USA. 
generically the eigenvalues go from purely imaginary to real (or vice versa). In case (ii), the sum of the eigenspaces of the eigenvalues $\pm i$ can be written as the sum of two $\omega$-orthogonal two-dimensional symplectic subspaces. This time, generically the eigenvalues go from purely imaginary into the right- and left-hand complex plane (or vice versa). We describe the behaviour of the eigenvalues in each of these cases by saying that the eigenvalues split, see figure 1. The 1-1 resonance with splitting is often called the Hamiltonian Hopf bifurcation, see [8].

It transpires that in many applications the eigenvalues do not behave in the manner described by the generic theory above. Rather than split at 0 or $\pm \mathrm{i}$, the eigenvalues remain on the imaginary axis and pass, see figure 2 . However it follows from the work of Galin [4], that at least three parameters are required for passing to be expected.

(a)

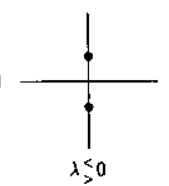

(b)

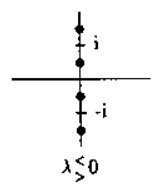

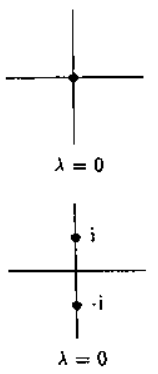



Figure 1. The splitting case: $(a)$ for the steady-state bifurcation; $(b)$ for the 1-1 resonance.

(a)

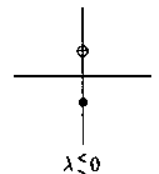

(b)

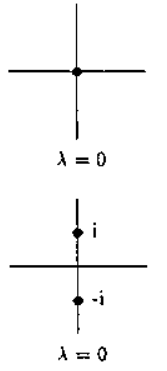

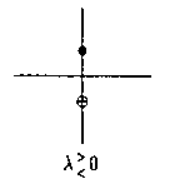

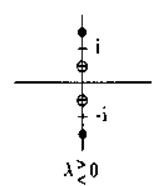

Figure 2. The passing case: (a) for the steady-state bifurcation; $(b)$ for the 1-1 resonance.

The reason that passing is seen so often in bifurcations of Hamiltonian vector fields is that in many applications there is symmetry present. As is well known in bifurcation theory (see for example [6]) the presence of symmetry can greatly influence the generic behaviour. Indeed, for certain symmetry groups (the most notable example being the circle group $S^{1}$ ), the passing of eigenvalues may be generic in a one-parameter family.

In the steady-state case, the dichotomy in eigenvalue movements can be understood using definiteness properties of the Hamiltonian, a method we shall call energetics, or group-theoretically (see Golubitsky and Stewart [5]). For the energetics method, see Oh [12] and for examples of the use of this criterion, see Oh et al [13]. We note that Krein theory primarily uses the energetics approach, but in a different way from that used in this paper. It turns out that energetics or group theory alone is not sufficient 
to characterize the movement of eigenvalues in the 1-1 resonance. One of the main purposes of this paper is to show that a combination of group theory and energetics yields a particularly clean characterization of the splitting and passing cases.

A more basic effect of the symmetry is to force multiplicity of certain eigenvalues, so that the dimensions given above for the various eigenspaces are often invalid. We prove results on the generic structure of the eigenspaces corresponding to the steady-state bifurcation and the 1-1 resonance (cf Golubitsky and Stewart [5, theorem 3.3] and van der Meer [9, p 1046]). Assume that the Hamiltonian is invariant under the symplectic action of a compact Lie group $\Gamma$. Theorem 3.2 states that in the case of a steady-state bifurcation, generically the generalized zero eigenspace $E_{0}$ is either non-absolutely irreducible or the direct sum of two isomorphic absolutely irreducible subspaces. (A $\Gamma$-invariant subspace $V$ is absolutely irreducible if the only linear mappings $V \rightarrow V$ that commute with the action of $\Gamma$ are real multiples of the identity. An irreducible subspace that is not absolutely irreducible is called non-absolutely irreducible.)

In the case of 1-1 resonance, theorem 3.3 states that generically the sum of the generalized eigenspaces of $\pm \mathrm{i}$, denoted by $E_{+\mathrm{j}}$, can be written as the sum of two symplectic $\omega$-orthogonal subspaces $U_{1}$ and $U_{2}$, where each of the $U_{j}$ is either non-absolutely irreducible or the direct sum of two isomorphic absolutely irreducible subspaces.

Although neither of these results is new, this is the first time that a complete proof has been given. (The proof in [5] of the first result contains non-trivial gaps, and the second result is stated but not proved in [9].)

The heart of the paper is concerned with the generic movement of eigenvalues in steady-state bifurcation and 1-1 resonance with symmetry. The steady-state bifurcation is well understood both group-theoretically [5] and in terms of energetics [12]. We combine these results in theorem 4.1. Recall that $E_{0}$ is generically either the direct sum of two isomorphic absolutely irreducible subspaces or is non-absolutely irreducible. These possibilities correspond precisely to the splitting or passing of eigenvalues. On the other hand, the linearization of the Hamiltonian vector field induces a quadratic form on $E_{0}$. This quadratic form changes from definite to indefinite in the splitting case but remains definite in the passing case.

The movement of eigenvalues in the $1-1$ resonance is rather more delicate. The results are summarized in theorem 4.4, see also table 1 . We show that it is necessary to combine the group-theoretic and energetic approaches in order to characterize the dichotomy, splitting or passing, in the eigenvalue movements. The interesting cases are when $U_{1}$ and $U_{2}$ are isomorphic, since if $U_{1}$ and $U_{2}$ carry distinct representations of $\Gamma$ then the resonance decouples and the eigenvalues move independently along the imaginary axis. In order to understand the cases where $U_{1}$ and $U_{2}$ are isomorphic, we make use of the results of Montaldi et al [10] on the interrelationship between the symmetry and the symplectic structure. At this stage it becomes necessary to distinguish between the two types of non-absolutely irreducible representations: complex and quaternionic. Provided $U_{1}$ and $U_{2}$ are not complex irreducibles, generically the eigenvalues split. This is accompanied by indefiniteness of the quadratic form induced on $U_{1} \oplus U_{2}$ by the linearization of the Hamiltonian vector field. If the $U_{j}$ are isomorphic complex irreducibles, then in the terminology of [10] they are either of the same type or dual. If $U_{1}$ and $U_{2}$ are complex of the same type, then generically the eigenvalues pass though the induced quadratic form is still indefinite. Finally, in the case of complex duals the eigenvalues can generically pass or split and these possibilities correspond precisely to definiteness and indefiniteness of the induced quadratic form. 
The paper is organized as follows. First, in section 2 we review the non-symmetric case. Using the Galin normal forms listed in [4], it is easy to verify that splitting is generic in the steady-state bifurcation and in the 1-1 resonance. We also describe the energetic viewpoint in this context.

In section 3 we formulate results on the generic structure of $E_{0}$ and $E_{ \pm \mathrm{i}}$ for a one-parameter family of linear Hamiltonian vector fields. Then, in section 4 we give group-theoretic and energetic characterizations of the movement of eigenvalues in the steady-state bifurcation. We also state and prove our main theorem, where we combine the group-theoretic and energetic methods to give a complete characterization of eigenvalue movements in the 1-1 resonance. We illustrate the results with some examples in section 5 .

Finally in section 6 , we discuss a possible source of confusion arising from the presence of additional symmetries when the Hamiltonian vector field is in Birkhoff normal form. We stress that our resuits are to be interpreted independently of any symmetries due to normal form so that $\Gamma$ consists only of external symmetries. Indeed, if normal form symmetries were included in $\Gamma$, then our results would become less sharp.

\section{The non-symmetric case}

In this section, we review the situation when there is no symmetry present. The results follow easily from work of Galin [4]. The codimension formula of Galin (see also Arnold [2, appendix 6]) implies that in a generic one-parameter family, each eigenvalue is precisely associated with one Jordan block of dimension at most two. Since zero eigenvalues of symplectic matrices have even multiplicity, it follows that in the steady-state bifurcation generically $\operatorname{dim} E_{0}=2$ and the restriction of the linearization is nilpotent. In the 1-1 resonance, by definition $\operatorname{dim} E_{ \pm i} \geq 4$ so generically this dimension is precisely four. Again, the restriction of the linearization is non-semisimple.

Let $A(\lambda)$ denote a one-parameter family of linear Hamiltonian vector fields undergoing one of these bifurcations at $\lambda=0$. In each case we can write $A(\lambda)$ in Galin normal form and explicitly compute the eigenvalues. The relevant normal forms in [4] are (36) and (35) respectively.

In the steady-state bifurcation, the Galin normal form of the linearized vector field contains the block

$$
M(\lambda)=\left(\begin{array}{ll}
0 & 1 \\
\lambda & 0
\end{array}\right) .
$$

The eigenvalues are given by $\pm \sqrt{\lambda}$, so as $\lambda$ increases through zero the eigenvalues move together along the imaginary axis and split onto the real axis.

In the 1-1 resonance, the Galin normal form with respect to the canonical symplectic structure contains the block

$$
M(\lambda)=\left(\begin{array}{cccc}
0 & -1 & \rho & 0 \\
1 & 0 & 0 & \rho \\
\lambda & 0 & 0 & -1 \\
0 & 0 & 1 & 0
\end{array}\right)
$$

where $\rho= \pm 1$. This time a computation yields the eigenvalues

$$
\pm \sqrt{\frac{1}{2}\{-(\rho \lambda+2) \pm \sqrt{\lambda(\lambda+8 \rho)}\}}
$$


In particular, for $\lambda$ close to 0 , the eigenvalues are purely imaginary precisely when the expression $\lambda(\lambda+8 \rho)$ is positive. Thus the eigenvalues split as required.

We now give a description in terms of energetics. An infinitessimally symplectic linear map $M$ on a symplectic space $Z$ induces a quadratic form $Q$ on $Z$ via the formula

$$
Q(z)=\omega(z, M z) .
$$

Moreover $Q(z)=\langle z, J M z\rangle$ so that the quadratic form is represented by the symmetric matrix $B=J M$.

Generalized eigenspaces are symplectic ([14] and proposition 3.7) and so we may speak of the quadratic form $Q$ induced on $E_{0}$ or $E_{ \pm \mathrm{i}}$ by $M(0)$. In the steady-state case we will denote by $Q_{\lambda}$ the quadratic form which is induced on the generalized eigenspaces of the eigenvalues going through 0 for $\lambda=0$. Note that $Q_{\lambda}$ is degenerate if and only if $M(\lambda)$ has a zero eigenvalue. In particular, in the case of 1-1 resonance, $Q$ is non-degenerate. In the steady-state bifurcation, $Q_{0}$ is degenerate, but $Q_{\lambda}$ is non-degenerate for $\lambda$ close but not equal to zero.

The following 'stability' theorem is a basic part of Krein theory, see Krein [7] and Moser [11].

Theorem 2.1 (Krein). Suppose that $M$ is an infinitessimally symplectic matrix defined on a symplectic vector space $Z$. Let $Q$ be the quadratic form induced on $Z$ by $M$. If $Q$ is definite, then $M$ is semisimple and the eigenvalues of $M$ lie on the imaginary axis.

Suppose that $A(\lambda)$ undergoes a steady-state bifurcation with $\operatorname{dim} E_{0}=2$. Then definiteness or indefiniteness of the quadratic form $Q_{\lambda}$ is governed by the sign (positive or negative) of det $B(\lambda)$. But in canonical coordinates, det $J=1$ so that $\operatorname{det} B(\lambda)=$ $\operatorname{det} M(\lambda)$. It follows that definiteness corresponds to purely imaginary eigenvalues and indefiniteness to real eigenvalues. Thus we have proved the following result.

Theorem 2.2. Suppose that a Hamiltonian system undergoes a steady-state bifurcation. Let $Q_{\lambda}$ denote the quadratic form induced on the corresponding generalized eigenspaces via equation (2.1). Then generically $\operatorname{dim} E_{0}=2$, and the eigenvalues move together along the imaginary axis and then split along the real axis (or vice versa). Simultaneously, the quadratic form $Q_{\lambda}$ changes from definite to indefinite (or vice versa).

The analogous result for the $1-1$ resonance is as follows:

Theorem 2.3. Suppose that a Hamiltonian system undergoes a 1-1 resonance. Let $Q$ denote the quadratic form induced on $E_{ \pm \mathrm{i}}$ via equation (2.1). Generically $\operatorname{dim} E_{ \pm \mathrm{i}}=4$, $Q$ is indefinite, and we have the splitting case.

Proof. It only remains to show that $Q$ is indefinite. But if $Q$ were definite, then by theorem 2.1 the eigenvalues would be constrained to lie on the imaginary axis and could not split.

\section{The generic structure of eigenspaces}

In this section, we describe the group-theoretic structure of the generalized eigenspaces $E_{0}$ and $E_{ \pm \mathrm{i}}$ in a generic one-parameter family of linear Hamiltonian vector fields with symmetry. In section 3.1 we state the main results; they are proved in section 3.2 . 


\subsection{Statement of results}

Let $Z$ be a symplectic vector space with symplectic form $\omega$. Assume that a compact Lie group $\Gamma$ is acting symplectically on $Z$, that is

$$
\omega(\gamma v, \gamma w)=\omega(v, w) \forall \gamma \in \Gamma \quad v, w \in Z .
$$

Let $\operatorname{sp}_{\Gamma}(Z)$ denote the Lie algebra of linear infinitessimally symplectic maps (or linear Hamiltonian vector fields) commuting with $\Gamma$ :

$$
B \in \operatorname{sp}_{\Gamma}(Z) \Longleftrightarrow\left\{\begin{array}{ll}
\text { (i) } & B: Z \rightarrow Z \text { is linear } \\
\text { (ii) } & \omega(B v, w)+\omega(v, B w)=0 \\
\text { (iii) } & \gamma B=B \gamma \quad \forall \gamma \in \Gamma .
\end{array} \quad \forall v, w \in Z\right.
$$

Suppose that $A$ is an element of $\operatorname{sp}_{\Gamma}(Z)$. Let $E_{0}$ and $E_{ \pm \mathrm{i}}$ denote the generalized eigenspaces of $A$ corresponding to the eigenvalues 0 and $\pm \mathrm{i}$ respectively.

In this paper we are primarily interested in the behaviour associated with $E_{0}$ or $E_{ \pm \mathrm{i}}$ that is generic, or to be expected, in a one-parameter family. However, the situation is non-trivial even when there are no parameters. A zero eigenvalue may be perturbed away so generically $E_{0}=0$. On the other hand, purely imaginary eigenvalues occur generically in the context of Hamiltonian systems. Moreover, these eigenvalues may have multiplicities forced by $\Gamma$ equivariance. Now we still have generically that $E_{ \pm \mathrm{i}}=0$ since we can simply scale the eigenvalues along the imaginary axis. However, it is convenient to disregard such scalings, since we can always normalize and bring the eigenvalues back to $\pm \mathrm{i}$. In this framework, it may be that $E_{ \pm \mathrm{i}}$ is non-trivial.

Theorem 3.1. Suppose that $A$ has an eigenvalue i. Then, disregarding the possibility of scaling the eigenvalue, generically either

(a) $E_{ \pm \mathrm{i}}$ is non-absolutely irreducible; or

(b) $E_{ \pm \mathrm{i}}=V \oplus V, V$ absolutely irreducible.

Now we can state our results for the case where $A$ is an element of a one-parameter family. It is now possible to have zero eigenvalues or resonant purely imaginary eigenvalues. Purely imaginary eigenvalues are in resonance when $E_{ \pm i}$ does not have one of the forms listed in theorem 3.1.

Theorem 3.2. Suppose that $A$ has a zero eigenvalue. Generically in a one-parameter family, either

(a) $E_{0}$ is non-absolutely irreducible; or

(b) $E_{0}=V \oplus V, V$ absolutely irreducible.

Theorem 3.3. Suppose that $A$ has a resonant eigenvalue i. Generically in a oneparameter family, $E_{ \pm \mathrm{i}}=U_{1} \oplus U_{2}$ where for $j=1,2$ either

(a) $U_{j}$ is non-absolutely irreducible; or

(b) $U_{j}=V \oplus V, V$ absolutely irreducible.

Remark 3.4(a). In theorem 3.1 the generalized eigenspace $E_{ \pm \mathrm{i}}$ is symplectic, that is $\omega_{\mid E_{t}}$ is non-degenerate (see proposition 3.7). Similarly $E_{0}$ is symplectic in theorem 3.2 . In theorem 3.3 the subspaces $U_{1}$ and $U_{2}$ may be chosen to be symplectic and also to be $\omega$-orthogonal. Recall that two subspaces $U_{1}$ and $U_{2}$ are $\omega$-orthogonal if $\omega\left(u_{1}, u_{2}\right)=0$ for all $u_{1} \in U_{1}$ and $u_{2} \in U_{2}$. 
(b) When there is no symmetry present, we may take $\Gamma$ to be the trivial group. The irreducible representations are absolutely irreducible and one-dimensional. Hence we recover the expected dimensions of the relevant generalized eigenspaces, as described in section 2 .

(c) The results in this section are proved in the following subsection using ad hoc techniques. Alternatively, they may be derived by generalizing Galin's codimension formula to the equivariant setting, see [15].

\subsection{Proofs}

3.2.1. Preliminaries. Let $\langle$,$\rangle be a \Gamma$-invariant inner product on $Z$. We may define a linear map $J: Z \rightarrow Z$ uniquely by

$$
\omega(v, w)=\langle v, J w\rangle \quad \text { for all } v, w \in Z
$$

Then $J$ is an isomorphism that commutes with $\Gamma$ and is skew-symmetric, that is $J^{T}=-J$. Conversely, given such a $J$, we may use equation (3.2) to define a symplectic form $\omega$ that satisfies (3.1).

A $\Gamma$-invariant subspace $W \subset Z$ that is symplectic is called $\Gamma$-symplectic. The restricted symplectic form $\omega_{\mid W}$ induces an isomorphism $J_{W}: W \rightarrow W$. Note that $J_{W}$ is not the same as $J_{\mid W}$. Indeed, $J$ will not in general leave the subspace $W$ invariant. Finally, we recall that a subspace $W \subset Z$ is isotropic if $\omega\left(w_{1}, w_{2}\right)=0$ for all $w_{1}, w_{2} \in W$.

We now state several basic results from Golubitsky and Stewart [5]. The first four results are theorem 2.1, proposition 2.3, proposition 3.1 and lemma 2.4 (b) of that paper. The fifth result is implicit in the proof of [5, theorem 2.1], although our choice of $J V$ is more constructive.

Proposition 3.5. $Z$ has the decomposition

$$
Z=\bigoplus U_{i}
$$

into $\Gamma$-symplectic $\omega$-orthogonal subspaces. Each $U_{i}$ is either non-absolutely irreducible or has the form $V \oplus V$ where $V$ is absolutely irreducible.

Proposition 3.6. If $E \subset Z$ is $\Gamma$-symplectic, then there is an $\omega$-orthogonal, $\Gamma$-symplectic complement $F$ so that $Z=E \oplus F$.

Proposition 3.7. The generalized eigenspaces of a linear map $A \in \operatorname{sp}_{\Gamma}(Z)$ are $\Gamma$ symplectic.

Proposition 3.8. If $V$ is $\Gamma$-irreducible, then $V$ is either isotropic or symplectic.

Proposition 3.9. (a) If $V$ is isotropic, then $V \cap J V=\{0\}$ and $V \oplus J V$ is $\Gamma$-symplectic.

(b) If $V$ is absolutely $\Gamma$-irreducible, then $V$ is isotropic. 
3.2.2. Proof of the theorems. We shall begin with the proof of theorem 3.2. Then the proofs of theorems 3.1 and 3.3 are similar. Almost all of this can be done in a coordinate-free setting. However, a computation is required to exclude certain possibilities in theorems 3.2 and 3.1 .

Lemma 3.10. Let $A(\lambda)$ be a one-parameter family in $\operatorname{sp}_{\Gamma}(Z)$ such that $A=A(0)$ has a zero eigenvalue. Generically either
(a) $E_{0}=V$
or
(b) $E_{0}=V \oplus J_{E_{0}} V$

where $V$ is a $\Gamma$-irreducible subspace of $\operatorname{ker} A$.

Proof. Since the kernel of $A$ is $\Gamma$-invariant it possesses an irreducible subspace $V$. If $V$ is symplectic, set $U=V$. Otherwise, $V$ is isotropic and we take $U=V \oplus J V$, (where $J=J_{E_{0}}$ ). In each case $U$ is $\Gamma$-symplectic and has an $\omega$-orthogonal, $\Gamma$-symplectic complement $Y$ in $E_{0}$ (propositions 3.7 and 3.8). Also, $E_{0}$ has an orthogonal symplectic complement $Z_{0}$ in $Z$. In symbols we have

$$
Z=E_{0} \oplus Z_{0} \quad E_{0}=U \oplus Y .
$$

If $Y=0$, there is nothing to do. Otherwise, define $B \in \mathbf{s p}_{\Gamma}(Z)$ in block-diagonal form as follows:

$$
\left.\dot{B}\right|_{U}=\left.B\right|_{Z_{0}}=\left.0 \quad B\right|_{Y}=J_{Y} .
$$

Set $A_{\epsilon}=A+\epsilon B$. Clearly, we have

$$
E_{0}\left(A_{\epsilon}\right) \subset E_{0} \quad V \subset \operatorname{ker} A_{\epsilon} .
$$

We claim that $E_{0}\left(A_{\epsilon}\right)$ is a proper subspace of $E_{0}$ for $\epsilon$ in a full deleted neighbourhood of zero. If the claim is true then we may proceed inductively until $Y=0$ thus proving the lemma. Note that $V$ is fixed throughout the induction, but in general $U$ may vary since $J=J_{E_{0}}$ depends on $E_{0}$,

It remains to verify the claim. Choose a non-zero vector $y \in Y$. It is sufficient to show that $y$ is not a generalized eigenvector corresponding to the eigenvalue 0 for all $\epsilon$ in a deleted neighbourhood of the origin. Suppose for contradiction that $A_{\epsilon}^{k} y=0$ for infinitely many $\epsilon$, where $k=\operatorname{dim} E_{0}$ say. Expanding $A_{\epsilon}^{k}$, we have

$$
\left(P_{k-1}(\epsilon)+\epsilon^{k} B^{k}\right) y=0
$$

for infinitely many $\epsilon$, where $P_{k-1}(\epsilon)$ is a matrix valued polynomial of degree $k-1$ in $\epsilon$. Equating components in the vector equation, and using properties of polynomiais, we see that equality holds for all $\epsilon$. Moreover, comparing coefficients of $\epsilon^{k}$, we have

$$
B^{k} y=0 \text {. }
$$

But $\operatorname{ker} B^{k} \cap Y=\{0\}$ so we have the required contradiction.

Proof of theorem 3.2. We must show that $V$ is generically non-absolutely irreducible in case (a) of the lemma and absolutely irreducible in case (b).

Non-absolute irreducibility in case (a) is automatic by proposition 3.9 since $E_{0}=V$ is symplectic and hence cannot be absolutely irreducible. Case (b) follows from a relatively tedious computation, see remark 4.7 . 
Proof of theorem 3.1. The proof is completely analogous to that of the previous lemma and theorem. This time we choose an irreducible subspace $V$ in the eigenspace of $\pm i$ and construct $U$ as before. Now write

$$
Z=E_{ \pm \mathrm{i}} \oplus Z_{0} \quad E_{ \pm \mathrm{i}}=U \oplus Y
$$

and replace $A_{\epsilon}^{k}$ by $\left[A_{\epsilon}^{2}+I\right]^{k}$ in the proof of the previous lemma.

Again $U$ cannot be absolutely irreducible. Also, generically it cannot be the direct sum of two non-absolutely irreducible subspaces, see remark 4.7.

Lemma 3.11. Let $A(\lambda)$ be a one-parameter family in $\operatorname{sp}_{\Gamma}(Z)$ such that $A=A(0)$ has a resonant eigenvalue i. Generically $E_{ \pm \mathrm{i}}=U_{1} \oplus U_{2}$ where $U_{1}$ and $U_{2}$ are symplectic $\omega$-orthogonal subspaces and for $j=1, \frac{1}{2}$ either
(a) $U_{j}=V$
or
(b) $U_{j}=V \oplus J_{U_{j}} V$

where $V$ is a $\Gamma$-irreducible subspace of the eigenspace of $\pm \mathrm{i}$.

Proof. The proof is similar to that of the previous lemma. This time we choose an irreducible subspace $V$ in the eigenspace of $\pm i$ and construct $U_{1}$ as before. By hypothesis, this is not the full generalized eigenspace, so we may construct a second symplectic subspace $U_{2}$. Write

$$
Z=E_{ \pm \mathrm{i}} \oplus Z_{0} \quad E_{ \pm \mathrm{i}}=U_{1} \oplus U_{2} \oplus Y
$$

and as before replace $A_{\epsilon}^{k}$ by $\left[A_{\epsilon}^{2}+I\right]^{k}$ in the proof of the previous lemma.

Proof of theorem 3.3. Again we must show that $V$ must be non-absolutely irreducible in case (a) and absolutely irreducible in case (b).

By construction, the $U_{j}$ are symplectic, so $V$ must be non-absolutely irreducible in case (a). It remains to consider the case $E_{ \pm i}=U_{1} \oplus U_{2}$ where $U_{1}$, say, is of type $V \oplus V$ and $V$ is non-absolutely irreducible. In fact we show that this case reduces to $U \oplus U$ where $U$ is of type (a). Begin by perturbing $U_{2}$ away as in the proof of the previous lemma. Then $E_{ \pm \mathrm{i}}=U_{1}$ is the sum of two isomorphic irreducible subspaces, so every irreducible subspace of $E_{ \pm \mathrm{i}}$ is isomorphic to $V$. By proposition 3.5 we may write $E_{ \pm \mathrm{i}}=U \oplus U$ where each copy of $U$ is symplectic and isomorphic to $V$. Hence this one copy of $U$ of type (b) in the lemma splits into two isomorphic copies of $U$ of type (a) in the theorem.

\section{Movement of eigenvalues}

Suppose that $A(\lambda)$ is a one-parameter family of linear Hamiltonian vector fields commuting with the action of a compact Lie group $\Gamma$. Suppose further that $A(\lambda)$ undergoes a steady-state bifurcation or 1-1 resonance at $\lambda=0$. Theorems 3.2 and 3.3 give the generic structure of the generalized eigenspaces $E_{0}$ and $E_{ \pm i^{*}}$

When there is no symmetry present, these structures reduce to those described in section 2. Moreover we were able to determine the generic movement of eigenvalues and to give an energetic description. In particular, the eigenvalues generically split off the imaginary axis in each bifurcation. 
When there is symmetry present, it is no longer true that the eigenvalues generically split. In this section, we show that the eigenvalues split off the imaginary axis or pass along the axis. Moreover this movement can be completely characterized in terms of group theory and energetics. In fact, in the steady-state bifurcation it is already known that the movement can be characterized using group theory alone [5] or by energetics alone [12]. We combine these two results in theorem 4.1.

The movement of eigenvalues in the 1-1 resonance is delicate and cannot be characterized by group theory alone or energetics alone. Even the statement of the result (theorem 4.4) requires familiarity with the terminology of Montaldi et al [10]. In section 4.1 we introduce this terminology and state theorems 4.1 and 4.4 . We also state some of the results in [10] that we shall require, including an equivariant version of Darboux's theorem. In section 4.2 we prove theorems 4.1 and 4.4 .

\subsection{Statement of results}

We begin by stating the combined results of [5] and [12] for the steady-state bifurcation.

Theorem 4.1. Suppose that the hypotheses of theorem 3.2 hold. Let $Q_{\lambda}$ denote the quadratic form induced on the corresponding generalized eigenspaces via equation (2.1). Generically, precisely one of the following occurs:

(a) $E_{0}$ is non-absolutely irreducible, $Q_{\lambda}$ is definite for $\lambda \neq 0$, and the eigenvalues pass with non-zero speed;

(b) $E_{0}$ is the direct sum of two isomorphic absolutely irreducible subspaces, $Q_{\lambda}$ changes from definite to indefinite, and the eigenvalues split.

In order to state the corresponding result for the 1-1 resonance, it is necessary to recall some terminology and results from Montaldi et al [10].

If $U$ is a symplectic representation then-by ignoring the symplectic structure-we obtain an ordinary representation, which is called the underlying representation. A $\Gamma$-irreducible symplectic representation is a representation that has no proper non-zero $\Gamma$-invariant symplectic subspaces. It follows from propositions 3.5 and 3.9 , part (b), that irreducible symplectic representations are either non-absolutely irreducible or the sum of a pair of isomorphic absolutely irreducible subspaces. Moreover, the following theorem holds, which is part of [10, theorem 2.1].

Theorem 4.2. (a) In the real and quaternionic cases the isomorphism type of the irreducible symplectic representation is uniquely determined by that of its underlying representation.

(b) In the complex case there are precisely two isomorphism types of irreducible symplectic representations for a given complex irreducible underlying representation. They are said to be dual to each other.

According to the two different possibilities occuring in part (b) we will speak of complex irreducibles of the same type and complex duals.

Remark 4.3. The real, complex and quaternionic cases mentioned in theorem 4.2 refer to the following well known fact (see, e.g., [6]): let $U$ be irreducible and $\mathscr{D}$ be the space of linear mappings $U \rightarrow U$ which commute with $\Gamma$. Then $\mathscr{D}$ is isomorphic to either $\mathbb{R}$, $\mathbb{C}$ or $\mathbb{H}$, where $\mathbb{H}$ denotes the quaternionics. Moreover, $U$ is absolutely irreducible if $\mathscr{D} \cong \mathbb{R}$ and non-absolutely irreducible if either $\mathscr{D} \cong \mathbb{C}$ or $\mathscr{D} \cong \mathbb{H}$.

After these preparations we state our main theorem of this section. 
Theorem 4.4. Suppose that the hypotheses of theorem 3.3 hold. Let $Q$ denote the quadratic form induced on $E_{ \pm i}$ via equation (2.1). Generically, precisely one of the following occurs:

(a) $U_{1}$ and $U_{2}$ are not isomorphic and the eigenvalues pass independently along the imaginary axis. ( $Q$ may be indefinite or definite.)

(b) $U_{1}=U_{2}=V \oplus V, V$ real, or $U_{1}=U_{2}=W, W$ quaternionic, the eigenvalues split, and $Q$ is indefinite.

(c) $U_{1}$ and $U_{2}$ are complex of the same type, the eigenvalues pass and $Q$ is indefinite.

(d) $U_{1}$ and $U_{2}$ are complex duals and the eigenvalues pass or split depending on whether $Q$ is definite or indefinite.

The statement of the last theorem is roughly summarized in table 1.

Table 1. Generic eigenvalue movement.

\begin{tabular}{llll}
\hline & Eigenspace structure & \multicolumn{2}{c}{ Induced quadratic form } \\
\cline { 3 - 4 } & & Definite & Indefinite \\
\hline (a) & $U_{1} \oplus U_{2}$ non-isomorphic & \multicolumn{2}{c}{ 'Independent passing' } \\
(b) & $V \oplus V \oplus V \oplus V$ real, or $W \oplus W$ quaternionic & Not generic & Splitting \\
(c) & $W \oplus W$ complex of the same type & Not generic & Passing \\
(d) & $W \oplus W$ complex duals & Passing & Splitting \\
\hline
\end{tabular}

Remark 4.5. (i) The case where there is no symmetry is contained in part (b) with $V$ real and $\operatorname{dim} V=1$.

(ii) Perhaps the most surprising aspects of theorem 4.4 are parts (c) and (d). In fact results of [10] explain why eigenvalues cannot split in part (c). There the so-called cyclospectral representations are classified. These representations have the property that all eigenvalues of commuting linear mappings lie on the imaginary axis. A representation of the form $U_{1} \oplus U_{2}$ where $U_{1}$ and $U_{2}$ are complex of the same type is indeed an example of a cyclospectral representation.

(iii) By extending the results of Galin to the equivariant setting [15], it is possible to prove theorem 4.4 in a less ad hoc manner. Moreover, the fact that passing occurs generically in the complex cases is seen to be transparent from this viewpoint.

Finally we state an equivariant version of Darboux's theorem, which is implicit in theorem 2.4 of [10]. We will use this result to simplify the computations involved in the proof of theorem 4.4 .

Proposition 4.6. Suppose that $U$ is an irreducible symplectic representation. Then, up iso isomorphism, there is precisely one symplectic form on $U$ in the real and quaternionic cases and precisely two in the complex case.

\subsection{Proofs}

Proof of theorem 4.1. Suppose that $E_{0}$ is non-absolutely irreducible. By remark 4.3 $A(\lambda)=a(\lambda) I$ where $a(\lambda) \in \mathscr{D}, \mathscr{D}=\mathbb{C}$ or $\mathbb{H}$, and $a(0)=0$. The eigenvalues of $A(\lambda)$ are the same as the eigenvalues of $a(\lambda)$ repeated with multiplicity equal to $\operatorname{dim}_{\mathscr{D}} E_{0}$. By proposition 4.6 we may choose coordinates so that $J= \pm \mathrm{i}$ (since these are candidates for $J$ and are distinct if $W$ is complex). The quadratic form $Q_{\lambda}$ is represented by the 
symmetric real matrix $B(\lambda)= \pm \mathrm{i} a(\lambda) I$. It follows that $a(\lambda)=\mathrm{i} b(\lambda)$ where $b(\lambda)$ is real. In particular, the eigenvalues of $A(\lambda)$ are purely imaginary. In addition, $a^{\prime}(0)=\mathrm{i} b^{\prime}(0)$ and $b^{\prime}(0)$ is generically non-zero, so that the eigenvalues pass through zero with non-zero speed. Finally $B(\lambda)$ is a real scalar multiple of the identity and so is definite for $\lambda \neq 0$ as required.

Now suppose that $E_{0}=V \oplus V$ where $V$ is real. By proposition 4.6 we may choose coordinates so that $J=\left(\begin{array}{cc}0 & -I \\ I & 0\end{array}\right)$. Again we may work with $2 \times 2$ matrices provided we include multiplicities equal to $\operatorname{dim} V$. But then we are back in the case where there is no symmetry and we can apply theorem 2.2 .

Proof of theorem 4.4. (a) Since $U_{1}$ and $U_{2}$ are non-isomorphic, there is a corresponding block diagonal structure of $A(\lambda)$ on $E_{ \pm \mathrm{i}}$ corresponding to the decomposition $E_{ \pm \mathrm{i}}=$ $U_{1} \oplus U_{2}$. The eigenvalues on each $U_{j}$ are simple up to multiplicities forced by symmetry and it follows that the eigenvalues belonging to each block remain on the imaginary axis and behave independently as $\lambda$ is varied. Similarly, the quadratic forms induced on the $U_{j}$ separately are definite. Depending on whether they are definite of the same sign or of opposite signs, the quadratic form on $E_{ \pm \mathrm{i}}$ is definite or indefinite.

(b) The case $U_{1}=U_{2}=V \oplus V$ reduces to the four-dimensional situation of theorem 2.3. We turn to the quaternionic case which is more difficult since we do not have a list of normal forms. Once we have verified that the eigenvalues split, it follows that $Q$ is indefinite by theorem 2.1. For simplicity from now on we suppress multiplicities forced by the dimension of the underlying irreducible representation, so we may reduce to the case where $W$ is isomorphic to $\mathbb{H}$ or even $\mathbb{C}^{2}$. Here it is convenient to use the standard representation of the quaternion $a+b i+c j+d k$ as the $2 \times 2$ complex matrix $\left(\begin{array}{c}a+b \mathrm{i} c+d \mathrm{i} \\ -c+d \mathrm{i} a-b i\end{array}\right)$. Choose coordinates so that $J(W) \subset W$ and that $J_{\mid W}=\left(\begin{array}{cc}0 & -1 \\ 1 & 0\end{array}\right)$.

On $E_{ \pm \mathrm{i}}$ the mapping $A(\lambda)$ has the form

$A(\lambda)=\left(\begin{array}{cccc}0 & a(\lambda) & u_{1}(\lambda)+\mathrm{i} u_{2}(\lambda) & v_{1}(\lambda)+\mathrm{i} v_{2}(\lambda) \\ -a(\lambda) & 0 & -v_{1}(\lambda)+\mathrm{i} v_{2}(\lambda) & u_{1}(\lambda)-\mathrm{i} u_{2}(\lambda) \\ -u_{1}(\lambda)-\mathrm{i} u_{2}(\lambda) & v_{1}(\lambda)-\mathrm{i} v_{2}(\lambda) & 0 & b(\lambda) \\ -v_{1}(\lambda)-\mathrm{i} v_{2}(\lambda) & -u_{1}(\lambda)+\mathrm{i} u_{2}(\lambda) & -b(\lambda) & 0\end{array}\right)$

where $a, b, u_{1}, u_{2}, v_{1}, v_{2}$ are real-valued functions. The computation of the eigenvalues of $A(\lambda)$ (using MATHEMATICA) leads to

$$
\sigma(\lambda)= \pm \frac{1}{\sqrt{2}} \sqrt{p(\lambda) \pm \sqrt{q(\lambda)}}
$$

with

$$
\begin{aligned}
p & =-\left(a^{2}+b^{2}+2\left(u_{1}^{2}-u_{2}^{2}+v_{1}^{2}-v_{2}^{2}\right)\right) \\
q & =p^{2}-4\left(a b-\left(u_{1}^{2}+u_{2}^{2}+v_{1}^{2}+v_{2}^{2}\right)\right)^{2} \\
& =\left((a-b)^{2}+4\left(u_{1}^{2}+v_{1}^{2}\right)\right)\left((a+b)^{2}-4\left(u_{2}^{2}+v_{2}^{2}\right)\right) .
\end{aligned}
$$

By assumption $\sigma(0)= \pm \mathrm{i}$ so $p(0)=-2, q(0)=0$. Since the first factor of $q$ is the sum of three squares, it is generically the case that the second factor vanishes. We compute at 0 that

$$
q^{\prime}=4\left((a+b)^{2}\right)^{\prime}-32\left(u_{2} u_{2}^{\prime}+v_{2} v_{2}^{\prime}\right)
$$


Now we claim that generically $q^{\prime}(0) \neq 0$ and hence we have the splitting case. It is clear that generically $q^{\prime}(0) \neq 0$ provided

$$
a(0) \neq-b(0) \quad \text { or } \quad u_{2}(0) \neq 0 \quad \text { or } \quad v_{2}(0) \neq 0 .
$$

Suppose that $a(0)=-b(0), u_{2}(0)=0$ and $v_{2}(0)=0$. We show that this is not a generic situation. The matrix $A(0)$ becomes

$$
A(0)=\left(\begin{array}{cccc}
0 & a & u & v \\
-a & 0 & -v & u \\
-u & v & 0 & -a \\
-v & -u & a & 0
\end{array}\right)
$$

and has semisimple eigenvalues $\pm \mathrm{i}\left(a^{2}+u^{2}+v^{2}\right)$.

Consider the perturbation

$$
A_{\epsilon}(0)=\left(\begin{array}{cccc}
0 & a+2 \epsilon & u+\mathrm{i} \epsilon & v \\
-a-2 \epsilon & 0 & -v & u-\mathrm{i} \epsilon \\
-u-\mathrm{i} \epsilon & v & 0 & -a \\
-v & -u+\mathrm{i} \epsilon & a & 0
\end{array}\right) .
$$

The eigenvalues $\sigma_{\epsilon}$ of this perturbed matrix are still purely imaginary,

$$
\sigma_{\varepsilon}= \pm \sqrt{-\left((a+\epsilon)^{2}+u^{2}+v^{2}\right)}
$$

but now we have generically that $q^{\prime}(0) \neq 0$ as required.

(c) By proposition 4.6 we may choose coordinates so that $J=\left(\begin{array}{ll}\text { i } & 0 \\ 0 & i\end{array}\right)$. Then $A(\lambda)$ has the form

$$
A(\lambda)=\left(\begin{array}{cc}
\mathrm{i} \alpha_{1}(\lambda) & a_{2}(\lambda) \\
-\frac{a_{2}(\lambda)}{1} \alpha_{4}(\lambda)
\end{array}\right)
$$

where $a_{2}$ is a complex-valued function and $\alpha_{1} ; \alpha_{4}$ are real. The eigenvalues are

$$
\sigma(\lambda)=p(\lambda) \pm \sqrt{q(\lambda)}
$$

where

$$
\begin{aligned}
& p=\frac{1}{2} \mathrm{i}\left(\alpha_{1}+\alpha_{4}\right) \\
& q=-\frac{1}{4}\left(\alpha_{1}-\alpha_{4}\right)^{2}-\left|a_{2}\right|^{2} .
\end{aligned}
$$

The eigenvalues of the matrix $B=J A(0)$ are

$$
\frac{1}{2}\left(-\left(\alpha_{1}+\alpha_{4}\right) \pm \sqrt{\left(\alpha_{1}-\alpha_{4}\right)^{2}+4\left|a_{2}\right|^{2}}\right)
$$

Generically in a one-parameter family $q(0)$ is non-zero. Hence by hypothesis on the eigenvalues we must have $p(0)=0$ and $q(0)=-1$. Therefore the eigenvalues of $A(\lambda)$ pass with non-zero speed provided $p^{\prime}(0)=\frac{1}{2} \mathrm{i}\left(\alpha_{1}^{\prime}(0)+\alpha_{4}^{\prime}(0)\right) \neq 0$. In addition $\alpha_{1}=-\alpha_{4}$ and so the eigenvalues of $B$ have opposite sign. 
(d) By theorem 4.2 and proposition 4.6 we may assume that $J=\left(\begin{array}{cc}i & 0 \\ 0 & -i\end{array}\right)$ and we have to investigate the eigenvalues of the matrix

$$
A(\lambda)=\left(\begin{array}{ll}
\mathrm{i} \alpha_{1}(\lambda) & a_{2}(\lambda) \\
a_{2}(\lambda) & \mathrm{i} \alpha_{4}(\lambda)
\end{array}\right)
$$

where again $a_{2}$ is a complex-valued function and $\alpha_{1}, \alpha_{4}$ are real. These eigenvalues are

$$
\sigma(\lambda)=p(\lambda) \pm \sqrt{q(\lambda)}
$$

where

$$
\begin{aligned}
& p=\frac{1}{2} \mathrm{i}\left(\alpha_{1}+\alpha_{4}\right) \\
& q=-\frac{1}{4}\left(\alpha_{1}-\alpha_{4}\right)^{2}+\left|a_{2}\right|^{2} .
\end{aligned}
$$

The eigenvalues of the matrix $B=J A(0)$ are

$$
\frac{1}{2}\left(\left(\alpha_{4}-\alpha_{1}\right) \pm \sqrt{\left(\alpha_{1}+\alpha_{4}\right)^{2}+4\left|a_{2}\right|^{2}}\right) .
$$

By assumption there are exactly two possibilities, both of which can be realized in a generic one-parameter family:

(i) $p(0)=0, q(0)=-1$. In this case the eigenvalues of $B$ are given by $-\alpha_{1} \pm \sqrt{\alpha_{1}^{2}-1}$ and $B$ is definite. Hence the eigenvalues of $A(\lambda)$ remain on the imaginary axis and pass with non-zero speed provided $p^{\prime}(0)=\frac{1}{2} \mathrm{i}\left(\alpha_{1}^{\prime}(0)+\alpha_{2}^{\prime}(0)\right) \neq 0$.

(ii) $|p(0)|=1, q(0)=0$. We claim that in this case generically $q^{\prime}(0) \neq 0$ and therefore we have the splitting case. We compute

$$
q^{\prime}(0)=-\frac{1}{2}\left(\alpha_{1}(0)-\alpha_{4}(0)\right)\left(\alpha_{1}^{\prime}(0)-\alpha_{4}^{\prime}(0)\right)+2\left|a_{2}(0) \| a_{2}\right|^{\prime}(0)
$$

and the eigenvalues are generically splitting at $\pm \mathrm{i}$ as long as

$$
\alpha_{1}(0) \neq \alpha_{4}(0) \quad \text { or } \quad a_{2}(0) \neq 0
$$

But the situation $\alpha_{1}=\alpha_{4}=1, a_{2}(0)=0$ can be perturbed to

$$
A_{\epsilon}(0)=\left(\begin{array}{cc}
\mathrm{i}(1+\epsilon) & \frac{1}{2} \epsilon \\
\frac{1}{2} \epsilon & \mathrm{i}
\end{array}\right)
$$

since $A_{\varepsilon}(0)$ still has the eigenvalue $\mathrm{i}\left(1+\frac{1}{2} \epsilon\right)$. Finally $B$ is indefinite since the eigenvalues split.

Remark 4.7. It is easily seen from the proof of theorem 4.4 that it is not generic in a one-parameter family for $A(0)$ to have only zero eigenvalues on the space $W \oplus W$ if $W$ is complex or quaternionic. For then the polynomials $p$ and $q$ both have to vanish yielding at least two independent conditions. This is the computation that was required to complete the proof of theorem 3.2. Similarly it is not generic for a matrix $A \in \operatorname{sp}_{\Gamma}(Z)$ to have resonant eigenvalues on the space $W \oplus W$, as required in theorem 3.1. 


\section{Examples}

(a) $\Gamma=S^{1} \times S^{1}$. We consider the following symplectic $S^{1} \times S^{1}$ action on $\mathbb{C}^{2}$,

$$
(\theta, \phi)\left(z_{1}, z_{2}\right)=\left(\mathrm{e}^{\mathrm{i} \theta} z_{1}, \mathrm{e}^{\mathrm{j} \phi} z_{2}\right)
$$

where the symplectic form $\omega$ is defined by $J=\left(\begin{array}{cc}\mathrm{i} & 0 \\ 0 & -\mathrm{i}\end{array}\right)$. The copies of $\mathbb{C}$ are nonisomorphic and therefore we know that splitting cannot occur.

For example, consider the $S^{1} \times S^{1}$-invariant Hamiltonian

$$
H\left(z_{1}, z_{2}, \lambda\right)=\frac{1}{2}\left(\lambda\left|z_{1}\right|^{2}-\left|z_{2}\right|^{2}\right)
$$

In real coordinates this takes the form

$$
H\left(q_{1}, q_{2}, p_{1}, p_{2}, \lambda\right)=\frac{1}{2}\left(\lambda\left(q_{1}^{2}+p_{1}^{2}\right)-\left(q_{2}^{2}+p_{2}^{2}\right)\right)
$$

$A(\lambda)$ is given by

$$
A(\lambda)=\left(\begin{array}{cc}
\mathrm{i} \lambda & 0 \\
0 & \mathrm{i}
\end{array}\right)
$$

and independent passing in a 1-1 resonance occurs for $\lambda= \pm 1$ whereas we have passing in the steady-state bifurcation case for $\lambda=0$.

Observe that $H$ is also invariant under the action of the transformations

$$
z_{1} \rightarrow \bar{z}_{1} \quad z_{2} \rightarrow \bar{z}_{2}
$$

but these transformations do not commute with $J$ and therefore act non-symplectically.

(b) $\Gamma=\mathrm{O}(2)$. We consider the symplectic form $J\left(z_{1}, z_{2}, z_{3}, z_{4}\right)=\left(-z_{2}, z_{1},-z_{4}, z_{3}\right)$ on $\mathbb{C}^{4}$. Let $\mathrm{O}(2)$ act on $\mathbb{C}$ by

$$
\theta \cdot z=\mathrm{e}^{\mathrm{i} \theta} z \quad \kappa \cdot z=\bar{z} .
$$

Then the diagonal action on $\mathbb{C}^{4}$ is symplectic and the copies of $\mathbb{C}$ are isomorphic real representations. Therefore we know by case (b) of theorem 4.4 that generically the splitting cases occur-with an eight-dimensional generalized eigenspace in the 1-1 resonance and a four-dimensional generalized eigenspace in the steady-state bifurcation.

For example, the Hamiltonian

$$
H\left(z_{1}, z_{2}, z_{3}, z_{4}, \lambda\right)=\lambda\left[\frac{1}{2}\left|z_{1}\right|^{2}-\operatorname{Re}\left(z_{1} \bar{z}_{2}\right)\right]-\left[\frac{1}{2}\left(\left|z_{2}\right|^{2}+\left|z_{4}\right|^{2}\right)+\operatorname{Re}\left(z_{1} \bar{z}_{4}\right)-\operatorname{Re}\left(z_{2} \bar{z}_{3}\right)\right]
$$

or in real coordinates

$$
\begin{aligned}
H\left(q_{1}, q_{2}, q_{3}, q_{4}, p_{1}, p_{2}, p_{3}, p_{4}, \lambda\right)=\lambda\left[\frac{1}{2}\left(q_{1}^{2}+q_{2}^{2}\right)-\left(q_{1} p_{1}+q_{2} p_{2}\right)\right] \\
-\left[\frac{1}{2}\left(p_{1}^{2}+p_{2}^{2}+p_{3}^{2}+p_{4}^{2}\right)+\left(q_{1} p_{3}+q_{2} p_{4}\right)-\left(q_{3} p_{1}+q_{4} p_{2}\right)\right]
\end{aligned}
$$




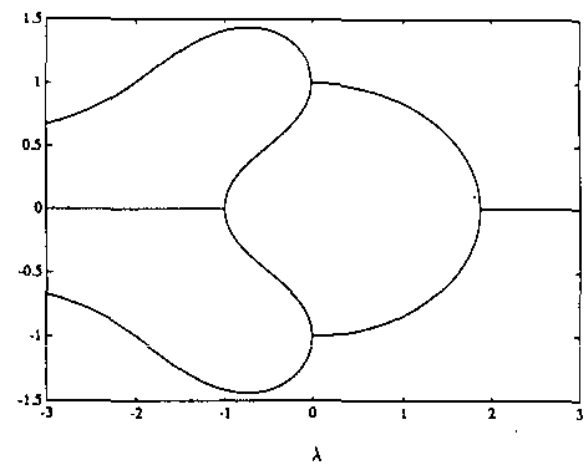

Figure 3. Imaginary parts of the eigenvalues in example (b) against the bifurcation parameter $\lambda$. The sequence splitting at $0 \rightarrow$ splitting at $\pm \mathrm{i}$ can be observed as $\lambda$ is varied. The last eigenvalue bifurcation is related to spliting off the real axis, but this does not correspond to a dynamic bifurcation.

is invariant under this $\mathrm{O}(2)$ action. For $-3 \leq \lambda \leq 3$ the movement of the four eigenvalues of multiplicity two is shown in figure 3 .

(c) $\Gamma=S^{1}$. We consider the space $\mathbb{C}^{2}$, where the symplectic form $\omega$ is induced by $J=\left(\begin{array}{ll}\mathrm{i} & 0 \\ 0 & \mathrm{i}\end{array}\right)$. A symplectic $S^{1}$-action on $\mathbb{C}^{2}$ is given by

$$
\theta\left(z_{1}, z_{2}\right)=\left(\mathrm{e}^{\mathrm{i} \theta} z_{1}, \mathrm{e}^{\mathrm{i} \theta} z_{2}\right) .
$$

Since the copies of $\mathbb{C}$ are complex of the same type, we have passing, generically with non-zero speed.

As an example we consider the $\Gamma$-invariant Hamiltonian

$$
H\left(z_{1}, z_{2}, \lambda\right)=\lambda\left[\frac{1}{2}\left|z_{1}\right|^{2}+\operatorname{Im}\left(\bar{z}_{1} z_{2}\right)\right]+\operatorname{Re}\left(z_{1} \bar{z}_{2}\right)
$$

which in real coordinates has the form

$$
H\left(q_{1}, q_{2}, p_{1}, p_{2}, \lambda\right)=\lambda\left[\frac{1}{2}\left(q_{1}^{2}+p_{1}^{2}\right)+\left(q_{1} p_{2}-q_{2} p_{1}\right)\right]+\left(q_{1} q_{2}+p_{1} p_{2}\right)
$$

Here $A(\lambda)$ is

$$
A(\lambda)=\left(\begin{array}{cc}
\mathrm{i} \lambda & \lambda+\mathrm{i} \\
-\lambda+\mathrm{i} & 0
\end{array}\right)
$$

and the eigenvalues of $A(\lambda)$ are $\mathrm{i} \lambda / 2 \pm \sqrt{-\left(\lambda^{2}+\frac{5}{4}\right)}$. A $1-1$ resonance occurs for $\lambda=0$ and the eigenvalues pass with non-zero speed.

(d) $\Gamma=S^{1}$. Again we consider the space $\mathbb{C}^{2}$, where the symplectic form $\omega$ is now induced by $J\left(z_{1}, z_{2}\right)=\left(z_{2},-z_{1}\right)$. The $S^{1}$ action on $\mathbb{C}^{2}$ is the same as in the last example, namely

$$
\theta\left(z_{1}, z_{2}\right)=\left(\mathrm{e}^{\mathrm{i} \theta} z_{1}, \mathrm{e}^{\mathrm{i} \theta} z_{2}\right)
$$

The spaces $\mathbb{C}\{z,-\mathrm{i} z\}$ and $\mathbb{C}\{z, \mathrm{i} z\}$ are complex duals. Therefore we generically have definite passing or indefinite splitting.

The following $\Gamma$-invariant quadratic Hamiltonian occurs in the analysis of a rotating orthogonal planar double pendulum as described in Bridges [3] (with $m=3$ )

$$
H\left(z_{1}, z_{2}, \lambda\right)=\frac{1}{8}\left|z_{1}\right|^{2}+\frac{1}{2} \lambda \operatorname{Im}\left(\bar{z}_{1} z_{2}\right)+2\left(1-\frac{3}{4} \lambda^{2}\right)\left|z_{2}\right|^{2} .
$$






Figure 4. Imaginary parts of all four eigenvalues in example (d) against the angular velocity $\lambda$. The sequence indefinite splitting $\rightarrow$ passing at $0 \rightarrow$ definite passing $\rightarrow$ passing at $0 \rightarrow$ indefinite splitting can be observed as $\lambda$ is varied.

In real coordinates

$$
H\left(q_{1}, q_{2}, p_{1}, p_{2}, \lambda\right)=\frac{1}{8}\left(q_{1}^{2}+q_{2}^{2}\right)+\frac{1}{2} \lambda\left(q_{1} p_{2}-q_{2} p_{1}\right)+2\left(1-\frac{3}{4} \lambda^{2}\right)\left(p_{1}^{2}+p_{2}^{2}\right)
$$

Here $\lambda$ corresponds to the angular velocity of the rotation. A more detailed study of this example will be performed in subsequent work. For $-2 \leq \lambda \leq 2$ the corresponding eigenvalue movement is shown in figure 4.

\section{Relationship to normal form theory}

It is well known that at 1-1 resonance there is an approximate phase shift $S^{1}$ symmetry introduced by the semisimple part of the linearization (see for example van der Meer [9]). More precisely, there are near-identity $\Gamma$-equivariant changes of coordinates so that the truncated Hamiltonian vector field (normal form) is $\Gamma \times S^{1}$-equivariant.

It can easily be shown that $E_{ \pm i}$ can be written as the sum of two isomorphic complex irreducible subspaces under the action of $S^{1}$. Moreover we have the following:

Proposition 6.1. Suppose that the hypotheses of theorem 3.3 hold. The decomposition of $E_{ \pm \mathrm{i}}$ into $\Gamma \times S^{1}$-irreducible subspaces is of the form $E_{ \pm \mathrm{i}}=U_{1} \oplus U_{2}$ where the $U_{j}$ are complex and are:

(1) non-isomorphic;

(2) of the same type; or

(3) dual.

Possibilities (1), (2) and (3) correspond to cases (a), (c) and (d) of theorem 4.4. In addition, case (b) of theorem 4.4 reduces to either possibility (2) or (3) above, and reduces generically to (3).

Proof. The proof is straightforward with the possible exception that case (b) of theorem 4.4 reduces generically to (3) rather than (2). But the decomposition in (2) is cyclospectral in the terminology of [10] and the eigenvalues must lie on the imaginary axis. By theorem 4.4 the eigenvalues generically split ruling out possibility (2). 
Remark 6.2. Although the proposition unifies some of the cases in theorem 4.4, it does not contain as much information as the theorem. Suppose that we are in case (b) of the theorem. Then, by the proposition we can generically pass to the case of complex duals by taking into account the additional $S^{1}$ symmetry. But then we can only conclude that passing or splitting occurs. However, by ignoring the $S^{1}$-symmetry, we are able to conclude that generically there is splitting.

\section{Acknowledgment}

We would like to thank Marty Golubitsky for very helpful discussions and suggestions.

\section{References}

[1] AbrahamR and Marsden J 1978 Foundations of Mechanics 2nd edn (New York: Addison-Wesley)

[2] Arnold V I 1989 Mathematical Methods of Classical Mechanics 2nd edn (Berlin: Springer)

[3] Bridges T 1990 Bifurcation of periodic solutions near a collision of eigenvalues of opposite signature Math. Proc. Camb. Phil. Soc. 108 575-601

[4] Galin D M 1982 Versal deformations of linear Hamiltonian systems AMS Transl. 2 118, 1-12 (1975 Trudy Sem. Petrovsk. 1 63-74)

[5] Golubitsky M and Stewart 1987 Generic bifurcation of Hamiltonian systems with symmetry Physica 24D $391-405$

[6] Golubitsky M, Stewart I and Schaeffer D 1988 Singularities and Groups in Bifurcation Theory vol 2 (Berlin: Springer)

[7] Krein M G 1950 A generalization of several investigations of A M Liapunov on linear differential equations with periodic coefficients Dokl. Akad. Nauk. SSSR $73445-8$

[8] van der Meer J C 1985 The Hamiltonian Hopf Bifurcation (Lecture Notes in Mathematics 1160) (Berlin: Springer)

[9] van der Meer J C 1990 Hamiltonian Hopf bifurcation with symmetry Nonlinearity 3 1041-56

[10] Montaldi J, Roberts M and Stewart I 1988 Periodic solutions near equilibria of symmetric Hamiltonian systems Phil. Trans. R. Soc. 325 237-93

[11] Moser J 1958 New aspects in the theory of Hamiltonian systems Commun. Pure Appl. Math. 9 81-114

[12] Oh Y-G 1987 A stability criterion for Hamiltonian systems with symmetry J. Geom. Phys. 4 163-82

[13] Oh Y-G, Sreenath N, Krishnaprasad P S and Marsden J E 1989 The dynamics of coupled planar rigid bodies. II. Bifurcations, periodic solutions, and chaos J. Dyn. Diff. Eqns 1 269-98

[14] Williamson J 1936 On the algebraic problem concerning the normal forms of linear dynamical systems Am. J. Math. 58 141-63

[15] Melbourne I and Dellnitz M Equivariant versions of the theorems of Williamson and Galin, in preparation 